\title{
Les origines de la ville d'Ypres (Xle-XIle siècles)
}

Adriaan Verhulst

\section{Citer ce document / Cite this document :}

Verhulst Adriaan. Les origines de la ville d'Ypres (Xle-XIle siècles). In: Revue du Nord, tome 91, n³29, Janvier-mars 1999. pp. 7-19;

doi : https://doi.org/10.3406/rnord.1999.2903

https://www.persee.fr/doc/rnord_0035-2624_1999_num_91_329_2903

Fichier pdf généré le 09/04/2018 
De oorsprong van de stad leper (11de-12de eeuw).

Net als Rijsel en in dezelfde periode, de tweede helft van de 10de eeuw, ontwikkelt leper zich als stad op een ruraal domein van de graaf van Vlaanderen. Die snelle groei is niet het gevolg van een grafelijke stichting, maar van een toevalsfactor, de overstroming van de Uzervlakte tijdens de eerste helft van de 1 Ide eeuw en de vor- ming van uitgebreide schorren. De daar geproduceerde schapenwol werd naar leper getransporteerd via een bijrivier van de IJzer, de leper, die weldra gekanaliseerd zou worden en bekend is als leperlee. In het grafelijke domein stonden aanvankelijk twee kerken, SintMaarten en Sint-Pieter. In een ervan - men weet niet dewelke (al ver- moedt de auteur dat het om Sint-Pieter gaat) - ontstond voor het einde van de 11de eeuw een kapittel van reguliere kanunnikken. De reformatorische bisschop van Terwaan, Jean de Warneton, schafte dit kapittel af wegens simonie. Hij stelde in Sint- Maarten een kapittel van reguliere kanunnikken in, in 1102, waarvan Sint-Pieter nu afhing, de nabijheid aldaar van een versterkte grafelijke residentie (Zaalhof) en de jaarmarkt, tenminste vanaf het begin van de 12de eeuw, voor de kerk, ten spijt. Bij Sint-Maarten werd de lokale markt gehouden waar de bevolking omstreeks 1127 vooral zeevis kon kopen. Op het einde van de 12de eeuw werd die uitgebreid en de beroemde hallen met een belfort werden het internationaal handelscentrum van de stad.

\begin{abstract}
The Origins of the City of leper (XI-XIlth centuries).

Just in the same way as Lille and around the same period, leper developed as a city through the second half of the XIth century on a rural estate owned by the Count of Flanders. This rapid growth has nothing to do with a deliberate foundation decreed by this latter but is due to an accidental factor, the flooding of the Yser plain during the first half of the XIth century and the consequent formation of vast salt meadows on which sheep were kept whose wool was transported towards leper on a tributary of the Yser, the leper, soon to be canalized under the name of the leperlee. The Count's estate originally had two churches, Saint-Martin's and Saint-Pierre's. In one, though it is impossible to determine exactly which (the author leans towards Saint- Pierre's), a chapter of secular cannons established itself before the end of the Xlthe century. This chapter was then abolished, owing to alleged cases of simony, by the reformist bishop of Therouanne, Jean de Warneton. He then establisched a chapter of regular cannons in Saint-Martin's in 1102, thus turning Saint-Pierre's into a subsidiary of Saint-Martin's, in spite of its proximity to a fortified residence of the Count (the Zaalhof) and the yearly fair that took place at its very foot as early as the beginning of the XIlth century at the latest. Close to Saint-Martin's there was the local market of the leper citizens who, around 1127, essentially bought seafood there. Around the end of the XIlth century this market expanded and the famous leper Halls with their belfrey made it the centre of the city international trade.
\end{abstract}

\title{
Résumé
}

Tout comme Lille et vers la même époque, Ypres s'est développée comme ville pendant la deuxième moitié du Xle siècle sur un domaine rural du comte de Flandre. Cette ascension rapide n'est pas due à une fondation délibérée de celui-ci mais à un facteur accidentel, à savoir l'inondation de la plaine de I'Yser pendant la première moitié du Xle siècle et la formation d'immenses prés salés dont la production de laine des moutons qui y étaient tenus était acheminée vers Ypres par la voie d'un affluent de I'Yser, l'leper, bientôt canalisé sous le nom d'leperlee. Le domaine comtal comptait à l'origine deux églises, Saint-Martin et Saint-Pierre. Dans l'une des deux, sans que l'on puisse déterminer laquelle (bien que l'auteur penche en faveur de Saint- Pierre), se constitua avant la fin du Xle siècle un chapitre de chanoines séculiers. Celui-ci fut supprimé sous prétexte de simonie par l'évêque réformateur de Thérouanne Jean de Warneton. II institua un chapitre de chanoines réguliers dans l'église Saint-Martin en 1 102, dont l'église Saint-Pierre devint une dépendance, nonobstant la proximité d'une résidence comtale fortifiée (le Zaalhof) et la tenue d'une foire annuelle à ses pieds dès le début du xne siècle au plus tard. Près de Saint-Martin d'autre part se tenait le marché local des Yprois qui autour de 1127 y achetaient surtout des poissons de mer. Vers la fin du xne siècle il fut agrandi et les fameuses halles d' Ypres avec le beffroi en firent le centre du commerce international de la ville. 


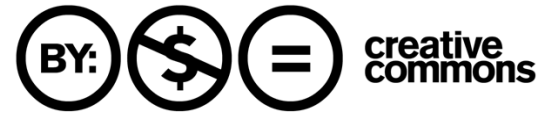




\section{ADRIAAN VERHULST}

\section{Les origines de la ville d'Ypres $\left(\mathrm{XI}^{\mathrm{e}}-\mathrm{XII}^{\mathrm{e}}\right.$ siècles $)$}

Bien qu'Ypres fût aux $\mathrm{XIV}^{\mathbf{c}}-\mathrm{XV} \mathrm{V}^{\mathbf{c}}$ siècles avec Gand et Bruges une des trois plus grandes villes du comté de Flandre' ${ }^{1}$, elle était d'origine beaucoup plus récente que ses deux rivales.

Alors que celles-ci remontaient aux $\mathrm{IX}^{\mathrm{C}}-\mathrm{X}^{\mathrm{C}}$ siècles, Ypres appartenait avec Saint-Omer et Douai d'une part et avec Lille d'autre part, à un groupe de villes du sud du comté dont la naissance se situe respectivement aux $\mathrm{X}^{\mathrm{e}}$ et $\mathrm{XI}^{\mathrm{c}}$ siècles ${ }^{2}$. Elle s'est développée, tout comme Lille et Douai, sur le territoire d'un grand domaine rural du comte et non pas en premier lieu, comme Gand ou Bruges, auprès ou en fonction d'une abbaye ou d'une fortification datant $\mathrm{du} I \mathrm{IX}^{\mathrm{C}}$ siècle. Son expansion a néanmoins été très rapide puisqu'un demisiècle seulement après le premier texte impliquant l'existence à Ypres d'un château comtal comme centre d'une châtellenie $(1066)^{3}$, la ville fut séparée du plat-pays environnant par l'octroi d'un droit particulier $(1116)^{4}$, tandis que s'y tenait très probablement déjà une foire annuelle, fréquentée en 1127 par des marchands étrangers et notamment italiens ${ }^{5}$.

1 - W. PrhthinitR, "La démographie des villes du comté de Flandre aux XIII" et XIV" siècles », Revue du Nord, t. 65, 1983, p. 255-275.

2 - A. DeRville, "La genèse et les premiers siècles de Lille ", dans La genèse et les premiers siècles des villes médiévales dans les Pays-Bas méridionaux. Actes du 14" Colloque de Spa 1988, Bruxclles, 1990, p. 247-279 (Crédit Communal. Collection Histoire in- $8^{\circ}, \mathrm{n}^{\circ} 83$ ); II)EM, Saint-Omer des origines au début du XIV' siècle, Lille, 1995; P. DEMOLON-E. LoUIS, "Naissance d'une cité médiévale flamande. L'exemple de Douai », dans P. DEMOLon e.a.(éds.), Archéologie des villes dans. le Nord-Ouest de l'Europe (VIr'-XIIr' siècle), Douai, 1994, p. 46-58.

3 - Charte du comte de Flandre Baudouin V pour le chapitre Saint-Picrre de Lille : in territorio Yprensi (F.L. GANSHOF, « Note sur une charte de Baudouin V, comte de Flandre, pour Saint-Picrre de Lille », Mélanges R. Crozet, Poitiers, 1966, p. 298-299). Le mot territorium a ici le sens de « châtellenie », dont le centre ćtait normalcment une fortification : cf. J. DHONDT, « Dévcloppement urbain et initiative comtale en Flandre au XI" sic̀cle », Revue du Nord, t. 30, 1948, p. I 44, n. 84 et p. 147.

4 - F. Vircauteren, Acres des comtes de Flandre 1071-1128, Bruxclles, 1938, n"79, p. 177-178. Cf. R. Van CAt:NiGilM, "Coutumes et législation en Flandre aux XI" et XII" sic̀cles ", dans Les libertés urbaines et rurales du Xr' au XIV' siècle. Colloque international Spa 5-8/IX/1966. Actes, Bruxelles, 1968 , p. 270 (Pro Civitate. Collection Histoire in- $8^{\circ}, n^{\circ} 19$ ).

5 - H. Pirennf: (éd.), Histoire du meurtre de Charles le Bon, comte de Flandre (1127-1128) par Galbert de Bruges, Paris, 1891, §16 (p. 28-29), § 20 (p. 35), §25 (p. 43). 
Les causes de cette ascension rapide n'ont jamais été élucidées de façon satisfaisante, bien que feu Jan Dhondt, dans le cadre d'une hypothèse hardie mais infirmée depuis lors, crût à une fondation de la ville et de la foire par le comte de Flandre Baudouin V (1035-1067) $)^{6}$. Selon M. Yamada, par contre, la foire d'Ypres n'a été au début qu'une des nombreuses foires que comptait la Flandre aux $X I^{\circ}-X I^{\circ}$ siècles. Ce n'est que vers la fin du XII' siècle que le cycle annuel des cinq foires de Flandre - Lille, Messines, Ypres, Torhout et Bruges - a éclipsé les autres foires flamandes (Gand et Courtrai notamment), probablement avec l'appui du comte. Mais toutes n'ont pas eu une origine comtale ${ }^{7}$. L'hypothèse de Dhondt que la fondation de plusieurs des cinq foires et de certaines des villes où elles se tenaient eût été accompagnée par la fondation d'un chapitre qui en constituait le support administratif, ne vaut certainement pas pour le chapitre de Saint-Martin à Ypres, comme nous l'avons démontré il y a quarante ans et comme nous le prouverons avec de nouveaux arguments ci-après ${ }^{\sharp}$.

Selon nos vues actuelles, en effet, la naissance d'une ville à Ypres pendant la deuxième moitié du $\mathrm{XI}^{\mathrm{c}}$ siècle et son ascension rapide ont été, pour une large part, l'effet indirect des grandes inondations qui en 1014 et de nouveau en 1042 ont submergé la vallée de l'Yser et toute la région entre Dixmude et Nieuport. A une quinzaine de kilomètres au nord d'Ypres, entre le cours actuel de l'Yser à l'est, qui remonte à cette époque, et la Oude Zeedijk («vieille digue de mer ») à l'ouest, construite tout de suite après ces inondations pour protéger la région de Furnes, celles-ci ont provoqué après quelques années, lorsque les eaux marines commençaient à se retirer, la formation d'immenses prés salés". Sur ceux-ci des textes nombreux signalent, à partir des années soixante du $\mathrm{XI}^{*}$ siècle, la présence de grands troupeaux de moutons. Leur exploitation était organisée dans le cadre d'unités appelées bercariae, dont on ignore malheureusement l'organisation interne ${ }^{10}$. On sait néanmoins qu'elles étaient données à cens pour des montants considérables payés en argent non monnayé, que la vente de la laine qu'elles produisaient a dû fournir ${ }^{\prime \prime}$. Celle-ci n'a donc vraisemblablement pas été travaillée sur place,

6- Dhonist, " Développement urbain » (voir n. 3), p. 147-149.

7 - М. Y.MMAD, "Le mouvement des foires en Flande avant 1200 », Mélanges Georges Despy, Liège, 1991, p. 773-789.

8 - A. VI:RHATST, «De vroegste geschiedenis van het Sint-Maartenskapittel en het ontstaan van de stad Ieper ", Handelingen vem de Maatschappij rour Geschiedenis en Oudheidkunde te Gent, nouvelle série t. I1, 1957, p. 31-48. Concernant Lille, l'hypothèse de Dhondt a été critiquée par

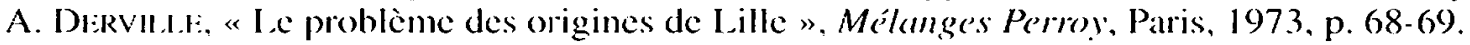

9 - A. Viskntist, « Lévolution de la plaine maritime flamande au Moyen Age ", Revtle de" I'Universite de Braxelles, 1962-1963, p. 89-106: In).M. Histoire du paysage rural en Flandre. Bruxelles, 1966, p. 25-29.

10 - Vercalitherin, Actes des comtes de Flandre..., p. 382, vo berquaria.

11 - Ibidem. p. XXVII, XXXIII-XXXIV, 13,14,160,176; A. Vherhul.st-M. Gysirina, Le Compte Général de 1187, comnu sous le nom de "Gros Brief" et le's institutions financières dut comté de Flandie all xIr siècle. Bruxelles, 1962, p. 142, 170, 181-183. Ct. A. Vt:RHULST, "Les biens et revenus du chapitre Saint-Donatien de Bruges en 1089 » in E. Moriter (éd.), Campagness 
mais probablement acheminée vers Ypres qui sur la terre ferme était l'endroit le plus proche des prés salés de la plaine inondée qu'on pouvait atteindre par une voie d'eau navigable et permanente. Ypres, en cffet, est situće sur le plus important des ruisseaux descendant des Monts de Flandre vers la vallée de l'Yser et qui se transformait en une rivière, appelée Iepere, à l'endroit où naîtra la ville à laquelle elle donna d'ailleurs son nom et où elle croisait la route importante venant de Paris par Lille et reliant à Bruges les cinq grandes foires de Flandre. A une quinzaine de kilomètres au nord d'Ypres cette rivière se jette dans l'Yser dont les eaux, par suite des inondations citées plus haut, ont dû rendre l'Iepere temporairement mieux navigable. Plus tard, après que les eaux des inondations se furent retirées et que la ville était née, on a canalisé l'Iepere, d'où le nom d'Ieperleet (leet signifiant «canal») que, depuis le $\mathrm{XII}^{\mathrm{C}}$ siècle et jusqu' aujourd'hui, elle porte encore ${ }^{12}$.

L'Yser lui-même, en aval du confluent avec l'Iepere où il entre dans la plaine d'inondation, devait être endigué pour rester navigable et trouver enfin son cours définitif vers la mer. En 1089, lorsqu'apparaît le nom de la future ville de Dixmude, à ce moment encore une dépendance de la paroisse rurale d'Esen et située à quelques kilomètres en aval du confluent de l'Iepere et de l'Yser, l'endiguement de l'Yser avait atteint cet endroit, dont le nom rappelle l'existence d'une digue (dic $)^{13}$. En 1163 ce travail était pratiquement achevé lorsque le comte Philippe d'Alsace fonda la ville de Nieuport (= nieuwpoort, «nouvelle ville») à l'embouchure de l'Yser dans la Mer du Nord. Une grande écluse y contrôlait l'écoulement des eaux des polders gagnés sur la plaine d'inondation de l'Yser et la nouvelle ville servait d'avant-port d'Ypres, succédant dans ce rôle à Dixmude ${ }^{14}$. La laine anglaise, que vers 1100 l'on avait commencé d'importer ${ }^{15}$ lorsque les prés salés au nord d'Ypres avaient été progressivement transformés en polders, ce qui réduisait sensiblement la production de laine indigène, y était débarquée. Acheminée vers Ypres elle devait assurer la continuation de l'approvisionnement en laine d'une industrie drapière qui s'était vraisemblablement implantée à Ypres

11. - (suite) médiévales : l'homme et son espace. Etudes offertes à Robert Fossier, Paris, 1995 , p. 513-531; IDEM, «Sheep-breeding and Wool Production in pre-thirteenth Century Flanders and their Contribution to the Rise of Ypres. Ghent and Bruges as Centres of the Textile Industry $\gg$ (sous presse dans Archeologie in Vlaanderen).

12 - O. Mus, «Van Sint-Pietersnederretting tot Sint-Pietersparochie », in Tien Eeuwen SintPietersparochie te Ieper; Ypres, 1991, p. 25-26.

13 - Sur Dixmude : A. Vishulst, Th. de Himplinnis, L. Di: Mi: , « Un tarif de tonlieu inconnu, institué par le comte de Flandre Thierry d'Alsace (1128-1168) pour le port de Littersuerua, précurseur du port de Damme », Bulletin de la Commission Royale d'Histoire CLXIV, 1998, p. 157-159. 14 - A. VhrHILST, «Initiative comtale et développement économique en Flandre au xII" siècle : le rôle de Thierry et de Philippe d'Alsace (1128-1191) », in Miscellanea Mediaevalia in memoriam J.F. Niemeyer, Groningue, 1967, p. 229-232.

15 - H. Van Werveke, «Essor et déclin de la Flandre », in Studi in onore di G. Luzatto, Milan, 1949. p. 154, réimpression dans IDEM, Miscellanea Mediaevalia, Gand, 1968, p. 5. 
dans la seconde moitié du $\mathrm{XI}^{\mathrm{e}}$ siècle en travaillant la laine des prés salés de la plaine d'inondation de l'Y ser $^{16}$.

Tandis que les facteurs géographiques et économiques dans les origines urbaines d'Ypres ont reçu peu d'attention dans l'historiographie traditionnelle, celle-ci est restée dominée par le problème que pose la présence simultanée à Ypres dès le début du XII ${ }^{\mathfrak{e}}$ siècle de deux églises. D'une part l'église Saint-Martin, considérée comme la plus ancienne des deux et aussi la plus importante à cause de son chapitre de chanoines, séculiers d'abord, puis réguliers ${ }^{17}$; d'autre part l'église Saint-Pierre, dont la première mention en 1102 se trouve dans le même document que celle de l'église Saint-Martin et où elle apparaît comme une dépendance de Saint-Martin, non pas avec le rang de chapelle mais comme église ${ }^{18}$. Saint-Pierre n'étant pas située au centre de la ville médiévale où se trouvent le Grand Marché, les halles et le beffroi et aussi l'église Saint-Martin, son origine a été expliquée par l'extension de la ville vers le Sud, où elle se trouve près de la porte de Lille. Entre ce dernier point et la place du marché le tracé régulier des rues, formé du Nord au Sud par trois rues parallèles et assez droites prenant leur départ au marché et croisées à des distances régulières par quelques rues parallèles en direction EstOuest, a été considéré comme un argument en faveur de cette extension de la ville et donc de l'origine plus récente de l'église Saint-Pierre"1". A ce propos quelques questions peuvent néanmoins être posées.

Comparant la location de la troisième église urbaine, Saint-Jacques (première mention $\left.1138^{2(}\right)$, près du Grand Marché, avec la distance qui sépare celui-ci de l'église Saint-Pierre, la position de cette dernière semble assez isolée. On en vient à se demander si la fondation d'une deuxième église urbaine à cet endroit, à une date assez précoce (1102 au plus tard) et le peuplement systématique d'un quartier neuf entre cette église et le marché, avec un parcellaire très régulier le long d'un bras de l'Iepere déjà canalisé à ce moment, sont des faits vraisemblables quelques dizaines d'années seulement après la naissance de la ville. Aussi certains historiens en sont venus à l'idée que Saint-Pierre pourrait être une église aussi ancienne ou même plus ancienne que Saint-Martin. Certains y ont vu une vieille église rurale ${ }^{21}$, mais d'autres,

16 - H. PIRl:N.vE, « Draps d’Ypres à Novgorod au commencement du XII" siècle », réimpression dans IDEM. Histoire économique de l'Occident médiéval. Bruges, 1951 p. 571-574.

17 - J. PYCKE. «Prévôté de Saint-Martin à Ypres », in Monasticon Belge, III. Flandre Occidentale. 3. Liège, 1974, p. 932-982.

18 - Charte de Jean de Warneton, évêque de Thérouanne, du 1 "octobre 1102, éd. E. Fi:ys-A.

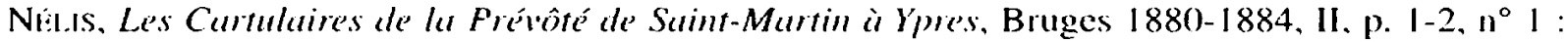
Prefatam e'rgo beati Martini ecclesiam eidem regendam tradidi, quan cum ecclesia beati Petri ceterisque suis appendiciis...liberam esse concessi.

19 - O. Mus, Van Sutstrata naar Rijselsestraat. Negen ecanen geschiedenis, Y pres, 1984.

20 - FEYs-Níls, Cartulaires de Saint-Martin..., p. 11-12, n 13.

21 - DHONDT, « Développement urbain », p. 149. 
notamment l'archiviste honoraire d'Ypres Octave Mus, la considèrent comme le centre d'un quartier commercial qui serait même plus ancien que le centre médiéval autour du Grand Marché ${ }^{22}$.

Le point de départ de l'hypothèse de Mus est le fait que la foire d'Ypres commençait le jour de la fête de la chaire de saint Pierre (in cathedra sancti Petri : 22 février). Un lien pourrait donc exister, selon lui, entre la foire et le saint patron de l'église. Ce serait notamment au pied de celle-ci que se serait tenue la foire. Galbert de Bruges, l'auteur d'un récit presque journalier des événements ayant suivi le meurtre du comte Charles le Bon à Bruges en 1127 et à qui l'on doit la première mention en cette année de la foire d'Ypres ${ }^{23}$, n'en donne cependant pas la localisation et de surcroît utilise le mot forum dans sa double acceptation de «foire» et de "place du marché ». Lorsque Galbert parle de ce dernier il ne le situe pas, mais précise que l'instrument avec lequel a été mis à mort le principal instigateur du meurtre, et qui n'était pas un gibet, se trouvait au milieu du marché « des Yprois » ${ }^{24}$. Cette locution particulière suggère qu'il y avait à Ypres, à part celui-ci, un autre marché, notamment pour les étrangers. Notre interprétation de cette distinction pourrait être confirmée par le fait, rapporté par Galbert, que sur le marché «des Yprois » se vendait du poisson ${ }^{25}$ et aussi par la présence sur le Grand Marché d'Ypres depuis le Moyen Age et jusqu' à la fin de l'Ancien Régime, d'un pilori que l'on peut considérer comme le successeur de l'instrument que Galbert situe sur le marché "des Yprois ${ }^{26}$. Ainsi on pourrait chercher, du moins à cette haute époque, le marché qui n'était pas spécialement destiné au commerce local, ailleurs dans la ville et notamment près de l'église SaintPierre. Cette localisation de la foire annuelle et internationale à une certaine distance du centre de la ville, aux confins de l'agglomération et même, comme c'était probablement le cas encore au début du XII' siècle, en dehors de celle-ci, correspondrait à la situation excentrique de beaucoup de foires internationales depuis le haut Moyen Age. Le Grand Marché d'Ypres d'autre

22 - O. Mus, «De stedelijke ontwikkeling van de Middeleeuwen tot 1914 », in Omtrent de vestingstad lepe'; Bruxelles. 1992, p. 4-13 (Fondation Roi Baudouin-Crédit Communal) et IDEM, « Van Sint-Pietersnederzetting » (cl. plus haut note 12).

23 - Voir plus haut note 5 .

24 - Galbert de Bruges. Histoire du meurtre de Charles le Bon, éd. Pirenne, \$ 57 (p. 92) : in medio fori Iprensium: $\$ 84(\mathrm{p} .129)$ : cum prepositus transiret per Ipram juxta patibulum in foro positum. 25 - Ibidem $\$ 57$ (p. 93): qui pro piscibus emendis in foro convenerant.

26 - P. DE: WIN, De schandstruffen in het wereldlijk strafiecht in de ZuidelijkeNederlanden, Bruxelles, 1991, n“- 127-129, 139 et 166. Cet auteur n'a toutefois pas analysé la description par Galbert de l'exćcution du prévôt Bertulphe et déclare donc (p. 157 note 8 ) à tort qu'il n'y a nulle part dans le récit de Galbert mention d'un pilori. Le récit de l'exécution par Galbert est confirmé par celui de Gautier de Thérouanne, Vita Karoli comitis, MGH, SS., XII, Hannovre, 1856, p. 554. Cet auteur ajoute un détail intéressant dans le contexte qui nous occupe en racontant comment le prévôt Bertulphe, en route vers le pilori, fut attaqué par des Yprois qui jetaient vers lui de grosses têtes de poisson de mer. Ce détail est à rapprocher de ce qui est dit à la note précédente (note 25) du marché où se vendaient des poissons. 
part a connu aux $X I^{*}$ et $X I I I^{\circ}$ siècles des transformations et des extensions qui lui ont donné seulement au bas Moyen Age son aspect définitif, conservé plus ou moins fidèlement à travers les âges ${ }^{27}$. En 1187 il s'étendait plutôt vers l'Est puisque le comte Philippe d'Alsace fit don en cette année d'un terrain faisant partie «de son marché » (in foro meo) pour y bâtir l'hôpital NotreDame qui se trouvait jadis à l'emplacement du palais de justice actuel, situé encore aujourd'hui à la limite orientale du Grand Marché ${ }^{2 x}$. D'autre part, une halle est signalée en 1173 qu'on peut vraisemblablement identifier avec la halle à la limite occidentale du Grand Marché cité dans un texte de 1247 (halla Yprensis). Une parcelle du terrain de la halle citée en 1173 avait appartenu auparavant à un particulier ${ }^{29}$. La construction de l'aile occidentale des halles actuelles peu avant 1286 a eu lieu également sur un terrain déjà divisé en parcelles individuelles ${ }^{30}$. La formation progressive du Grand Marché d'Ypres sur des terrains non vierges ressemble ainsi à la création de la Grand' Place à Lille (actuellement Place du Général de Gaulle) et du Marché du Vendredi à Gand au XIII' siècle, qui dans ce dernier cas a nécessité la destruction de maisons situées à cet endroit ${ }^{31}$.

Une donnée topographique d'un autre ordre suggère également l'aménagement auX XII et XIII' siècles du quartier à l'ouest des halles au détriment des environs de Saint-Pierre.

Au cours du XII" siècle probablement, un canal nommé Scipleet a été creusé dans la ville parallèlement à la rivière qui depuis lors s'appelait Viese (vieille) Ypre. Depuis le point où celle-ci sort de la ville, à l'ouest des halles, ce canal, en amont, c'est-à-dire en direction du Sud et vers l'intérieur de la ville, ne court pas plus loin qu'à mi-chemin de la rue parallèle (appelée jadis Zuudstrate, actuellement « rue de Lille » = Rijselse stract), qui part du Grand Marché au nord vers la porte de Lille au sud ${ }^{32}$. L'important du point de vue qui nous occupe c'est que ce canal n'a pas été creusé jusqu'à hauteur de l'église Saint-Pierre. Sur la vieille Iepere, près de l'église Saint-Pierre, se trouvait par contre encore plus tard une «porte d'eau » (anc. flamand over-

27 - (). Mus, « De geboorte van een marktplein ", in De leperse markt, e'en historisch fenomeen, Ypres, 1985, p. 3-27 (publication de la Kredietbank).

28 -.. Ii:YS-Nisis. Cartulaires Saint-Martin.... n 41, p. 29.

29 - C.B. Di: RIDDER, «Documents extraits du cartulatire de Grimbergen », Analectes pour semvir à Ihistoire ecclésiastique de la Beigique, t. XI, 1874, p. 17. Labbaye de Girimbergen avait acquis les revenus d'une parcelle dans la halle: en 1247 elle vendit ses revenus à Ypres à Saint-Martin d'Ypres, notamment une rente assignée sur la halla Yprensis (FEys-Nitds, Cartulaires Saint-Martin..., II, p. $109-110)$.

30 -..- Mis, “Gcboortc marktplein...", p. 16, sur base d'un regeste conservé dans Bibliothèque Royale, Fonds Merghelynck, ms. 90, III, p. 604.

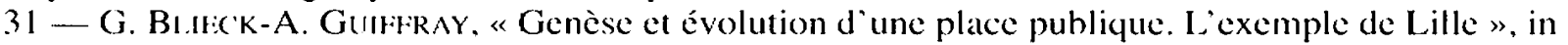
P. Dimolon, H. Galinie, F. Vhrhatiht (éds.). Archéclogic dés villes dans le Nord-Ouest de l'Europe (vIr-XIIr siècle), Douai, 1994, p. 204-224, spéc. p. $219-221$.

32 - Mus, Van Sutstrata (sans pagination). 
dragh, néerl. overtoom) ${ }^{33}$. Elle doit dater, compte tenu de la canalisation de l'Iepere en aval, à l'intérieur de la ville, par le creusement parallèle de la Scipleet, de l'époque où la rivière était encore navigable jusqu'à l'intérieur de la ville, par suite des inondations de la vallée de l'Yser, c'est-à-dire du XI ${ }^{\mathrm{e}}$ ou du début du XII" siècle. La construction de l'overdragh près de Saint-Pierre fut donc vraisemblablement antérieure à cette canalisation. Celle-ci, par contre, semble avoir été réalisée au profit du quartier du Grand Marché et plus particulièrement de l'extension vers l'Ouest de celui-ci lors de la construction des halles dans la deuxième moitié du XII' siècle. La situation de la porte d'eau près de l'église Saint-Pierre constitue donc un argument en faveur du caractère commercial du quartier autour de Saint-Pierre, antérieurement à l'extension du Grand Marché vers l'Ouest. Pour confirmer cette hypothèse, due à Octave Mus $^{34}$, et localiser la foire internationale près de l'église Saint-Pierre, on dispose enfin d'un argument d'un tout autre ordre.

Il s'agit de la proximité de la résidence comtale appelée Zaalhof, située à hauteur de l'église Saint-Pierre, mais sur la rive opposée, donc occidentale, de l'Iepere. Cette proximité pourrait être interprétée comme témoin de la protection accordée par le comte à la foire et ressembler à la situation de la foire de Torhout, entre Ypres et Bruges, où dès avant la fin du XI $\mathrm{l}^{\mathrm{e}}$ siècle une fortification comtale protégeait la foire qui au $\mathrm{XII}^{\mathrm{c}}$ siècle fera partie du cycle des cinq grandes foires flamandes parmi lesquelles celle d'Y pres était une des plus importantes ${ }^{35}$. Toutefois le Zaalhof a été considéré jusqu'à présent par les historiens d'Ypres comme une construction nouvelle de la fin du $\mathrm{XII}^{\circ}$ siècle, ce qui cadre mal avec une localisation de la foire dans ses environs un siècle plus tôt, avant le transfert de celle-ci dans les halles ${ }^{36}$. Cette datation tardive repose principalement sur la construction vers $1187 / 1190$ d'une chapelle dans le Zaalhof ${ }^{37}$. L'exemple de la construction d'une chapelle dans le "Château des Comtes" à Gand, quelque temps seulement après la construction en 1180 du château lui-même, enlève toutefois du poids à cet argument ${ }^{38}$.

\footnotetext{
33 - Fi:ys-NF́ı,IS, Cartulaires Saint-Martin.... n 353, p. 257-258 : d'encoste l'overdragh à Saint Pierre sour l'Ypre (1296). Cl. H. PIRFNNE, « Les "overdraghes" et les "portes d'eau" en Flandre au XIII" sic̀cle, à propos d'une charte inćdite des archives de la ville d'Ypres ", réimpression dans IDis, Histoire économique de l'Occident médiéval..., p. 542-550. En dehors de la villc, au nord de celleci, la navigabilité de l'Iepere jusqu'à l'Yscr était assurée, après la période des inondations (première moitié du XI" siècle), par plusicurs de ces constructions. Voir la carte du XVIII" siècle reproduite dans A. VerhuLST, Landschap en landbouw in middeleeuws Vlaanderen. Bruxclles, 1995, p. 114. 34 - Voir ses articles cités plus haut, notes 12 et 19.

35 - YAMADA, « Le mouvement des foires...», p. 780-781.

36 - DHONDT, «Initiative comtale », p. 147-149; VERHULST, « De vrocgste geschiedenis van het Sint-Maartenskapittel...».

37 - FEYS-NÉLIS, Cartulaires Saint-Martin..., n²6, p. 19-20.

38 - Charte du comte Baudouin IX de 1201, éd. W. PrevenIER, De oorkonden der graven van! Vlaanderen (1191-aanvang 1206), II, Uitgave, Bruxclles, 1964, n 169, p. 369.
} 
Le caractère récent du Zaalhof ressortirait néanmoins aussi, selon ces mêmes historiens ${ }^{39}$, de l'existence d'une fortification comtale plus ancienne dont il aurait pris la relève et située ailleurs, notamment à l'ouest du Grand Marché, plus précisément entre l'église Saint-Martin et la vieille Iepere. Le bras canalisé de celle-ci, la Scipleet, se détache de la vieille Iepere juste au nord de la localisation hypothétique de cette fortification, de sorte que celleci fût entourée d'eau par trois côtés. On ne dispose cependant pas de données écrites ou archéologiques sur l'existence à cet endroit d'une fortification, bien que la mention en 1066 d'un territorium Yprense, c'est-à-dire d'une châtellenie, implique l'existence d'un tel ouvrage quelque part dans ou près de la ville naissante ${ }^{40}$. La toponymie révèle l'existence d'une motte à cet endroit, mais ne réfère pas à son propriétaire ${ }^{41}$, contrairement aux toponymes concernant la résidence comtale du Zaalhof qui l'appellent le mote le conte et sgravenwal aux XIII' et XIV siècles, avant l'introduction au XVI ${ }^{\mathfrak{c}}$ siècle du nom Zaalhof ${ }^{42}$. Néanmoins les recherches archéologiques et topographiques de J. Termote en 1990 auraient démontré l'existence de deux fossés circulaires dont l'un aurait même entouré l'église Saint-Martin et l'autre, situé à l'ouest du premier, une motte ${ }^{43}$. Bien qu'il n'existe plus aucune trace archéologique de cette motte, Termote pense pouvoir reconnaître sur le plan cadastral du $\mathrm{XIX}^{\mathrm{C}}$ siècle ainsi que sur une photo aérienne de la ville après sa destruction presque totale pendant la première guerre mondiale, le tracé d'un demi-cercle qu'on pourrait interpréter comme la partie occidentale du fossé ayant entouré la motte. Celle-ci aurait donc été peu après le milieu du $\mathrm{XI}^{\mathrm{c}}$ siècle la fortification primitive du comte à Ypres. Elle aurait été abandonnée au profit du Zaalhof à une date qu'on pourrait situer avant la première mention de la foire en 1127 si le lien entre celle-ci et le Zaalhof que nous avons supposé pouvait être établi avec certitude.

Quoi qu'il en soit, la présence d'une première fortification comtale auprès de l'église Saint-Martin renforce à première vue l'opinion traditionnelle qui voit en elle une église du comte, telle qu'on en rencontre comme collégiale auprès des résidences comtales primitives dans les grandes villes

39 - Voir ci-devant note 36 et aussi le travail ancien mais toujours valable de G. DES MARE\%, Etude sur la proprićté foncière dans les villes du moyen âge et spécialement en Flandre, Gand-Paris, 1898, p. 203-2()4.

40 - Voir plus haut note 3.

41 - Le mote devant le paradis a Sainct Martin (1288-1309): gisant devant latrie St Martin sour le Scipleet sous le angle dele ruele dele mote vers zund (avant 1317): vall der plache up den wal voor St Martins (avant 1329) : voir VeRHuLST, «Vroegste geschiedenis St Maartenskapittel...», p. 3, note 15 ; J. THRMOTE, « De Sint-Maartenskerk en de vroegste stadsontwikkeling van leper », dans $D e$ Sint-Maartenskathedraal te leper, Ypres, 1990, p. 84, note 11.

42 - A le mote du comte, sour le viese Ypre (1320); tender Nederstrate tsinte Pieters also men gaet sgravenwalle waert (1431). Voir FEYS-NÉL.IS, Cartulaires Saint-Martin..., $\mathrm{n}^{* 3} 397$ et 816.

43 - TERMOTE, «De Sint-Maartenskerk...»; IDEM, « Het stadsarcheologisch onderzoek te Ieper in 1988-1989», Westvlaamse Archaeologica, t. 6, 1990, n³. 
flamandes ${ }^{44}$. Toutefois Saint-Martin d'Ypres n'était pas, en tant que chapitre de chanoines, une fondation du comte, contrairement à la thèse défendue jadis par feu Jan Dhondt ${ }^{45}$. Aux arguments que de son vivant nous avons fait valoir contre la thèse de Dhondt ${ }^{46}$, d'autres peuvent aujourd'hui être ajoutés.

En 1102 l'évêque réformateur de Thérouanne, Jean de Warneton, transforma Saint-Martin en une abbaye de chanoines réguliers selon la règle de saint Augustin, comme il l'a fait avec de nombreuses églises de son diocèse ${ }^{4 \text { ? }}$. Aucune de ces réformes n'a cependant eu lieu dans un chapitre comtal et y aurait d'ailleurs été impensable ${ }^{48}$.

En deuxième lieu la pauvreté de l'abbaye de Saint-Martin pendant tout le $\mathrm{XII}^{\mathfrak{e}}$ siècle, comparée à la richesse des chapitres comtaux de Bruges, Lille ou même Cassel, ne manque pas de frapper. Les comtes flamands du XII siècle n'ont fait aucune donation à Saint-Martin, à part l'octroi de l'immunité judiciaire sur les quelques biens à Ypres que l'église avait reçus de certains particuliers $^{49}$. Même Guillaume d'Ypres, petit-fils du comte Robert le Frison, bien que la base de son pouvoir éphémère se trouvât à Ypres, notamment lors des événéments ayant suivi en 1127-1128 le meurtre du comte Charles le Bon auquel il voulut succéder ${ }^{50}$, n'a pas fait bénéficier Saint-Martin d'Ypres de la générosité qu'il montra envers l'abbaye de Lo, située au milieu de son domaine familial à une vingtaine de kilomètres au nord d'Ypres. Celle-ci non plus ne reçut aucun don des comtes de Flandre du XII ${ }^{c}$ siècle, ce qui n'est peut-être pas sans signification dans le contexte des relations troublées entre le comte et ce seigneur temporairement rival et puissant dans la région d'Ypres. L'histoire ecclésiastique de l'église de Lo aussi ressemble d'ailleurs à celle de Saint-Martin d'Ypres, puisqu'elle fut également transformée vers la même époque par l'évêque Jean de Warneton en une abbaye de chanoines réguliers selon la règle de saint Augustin ${ }^{51}$. Cette réforme a même eu dans les deux églises une cause identique, à savoir le fait que les clercs qui s'étaient groupés en chapitre séculier aussi bien autour de l'église de Lo que dans celle

44 - A. VERHULST, « Dic gräfliche Burgenverfassung in Flandern im Hochmittelalter », dans H. PATZE, Die Burgen im deutschen Sprachraum, I, Sigmaringen, 1976, p. 277-278 (Vorträge und Forschungen XIX).

45 - DHONDT, « Développement urbain...», p. 147-149.

46 - Vi:RHUL.ST, «Vrocgste geschiedenis van het Sint-Maartenskapittel...».

47 - PrCKE, «Prévôté de Saint-Martin...» (voir plus haut note 17), p. 939-940; M. CARNIER, «Jan van Waasten en de hervorming van het Iepersc kapittel », in W. Verbeke e.a. (éds.), Serta devota in memoriam Guillelmi Lourdeaux, Louvain, 1995, p. 57-64.

48 - G. DECLliRCQ, « Seculiere kapittels in Vlaanderen », De Leiegouw, t. 28, 1986, p. 235-242; IDEM, « Het kapittel van Harelbeke in de 11 e en 12e eeuw », Sacris Erudiri, t. 29, 1986, p. 269-312, spéc. p. 283.

49 - PYCKr:, "Prévôté Saint-Martin...», p. 941. Cf. la charte de Philippe d'Alsace de 1187, citée plus haut note 28 : quia huius ecclesie canonici pancos admodum redditus habentes.

50 - E. WARloP, «Willem van Ieper. Een Vlaams condottiere », De Leiegouw, t. 6, 1964, p. 167191 et 7,1965, p. 197-218.

51 - J. PYCKE, «Prévôté puis abbaye de Saint-Pierre à Lo », dans Monasticon belge, III, Flandre Occidentale, 3, Liège, p. 891-893. 
d'Ypres, auraient obtenu par simonie le tiers de la dîme (altare) de leur église $^{52}$, alors que les deux tiers (bodium) se trouvaient depuis longtemps aux mains d'une autre église, notamment celle de Saint-Donatien de Bruges depuis au moins 1089 en ce qui concerne le bodium d'Ypres et cela vraisemblablement grâce à une donation du comte de Flandre ${ }^{5.3}$.

Alors que la réforme à Lo connut un déroulement sans incidents, celle de l'église d'Ypres fut marquée par diverses péripéties ${ }^{54}$. Les chanoines séculiers, en effet, s'y opposèrent et leur chef, un certain clerc Baudouin, alla plaider leur cause à Rome. Le pape chargea l'évêque d'Arras de trouver une solution au problème de la possession de l'altare. Après encore quelques manoeuvres dilatoires des chanoines, celui-ci, avec l'accord du pape, donna raison en ce qui concerne la simonie à son collègue de Thérouanne Jean de Warneton, qui chassa les chanoines de l'église. L'évêque, au lieu de procéder immédiatement à la réforme et d'ouvrir l'église à nouveau au culte, retarda la réforme pendant probablement quelques mois en gardant l'église pardevant lui. Toutefois, sous la pression de quelques «bonnes gens » (bonorum virorum) d'Ypres, l'évêque fit enfin procéder à l'élection du chef du chapitre réformé et lui transmit l'église de Saint-Martin avec celle de Saint-Pierre et autres dépendances de Saint-Martin. La charte épiscopale du $1^{\text {*r }}$ octobre 1102 , qui fait connaître ces péripéties ${ }^{55}$, est le premier document dans lequel deux églises sont citées à Ypres et, pour la première fois également, avec le nom du saint patron de chacune. Avant cette date et encore au début de la charte épiscopale elle-même, il est question dans les documents, sans autre spécification, de l'église «d'Ypres» (ecclesia Iprensis), du nommé Baudouin comme clericus Iprensis, des clerici Iprenses, de l'altare Iprense et en 1089 du bodium de Hipris. Cette formulation doit vraisemblablement être interprétée comme la preuve de ce qu'il n'y avait à Ypres avant 1102 et encore en cette année même, qu'une seule église possédant les pleins droits paroissiaux.

Jusqu'à présent on a toujours identifié celle-ci, même avant 1102, avec Saint-Martin. La raison principale de cette identification était toutefois la situation à partir de 1102, lorsque Saint-Martin faisait fonction d'église principale, Saint-Pierre n'étant qu'une dépendance de celle-ci, malgré le titre d'ecclesia et non de capella que lui confère la charte épiscopale de 1102. Les péripéties de la réforme et les indices d'une certaine ancienneté de l'église Saint-Pierre, exposés plus haut, nous amènent cependant à croire que ces péripéties peuvent avoir eu pour cause non seulement l'existence de l'église Saint-Pierre avant 1102, mais peut-être aussi un statut ecclésiastique plus important que celui d'une simple dépendance. Une légende yproise de la fin

52 - PYCKe, «Prévôté Saint-Pierre à Lo...», p. 892 ; IblM, «Prévôté Saint-Martin à Ypres...», p. 939 ; CARNiER, «Jan van Waasten...». p. 61.

53 - Charte du comte Robert Il du 31 octobre 1089. éd. M. Gyssti.INGi-A.C.F. KoCH, Diplomata belgica ante annum 1100 scripta, s.1., 1960, n 170, p. 297 : Bodium de Hipris.

54 - Exposées en détail par CARNIER, « Jan van Waasten...».

55 - Fi:YS-Nélis, Cartulaires Saint-Martin..., n 1, p. 1-2. 
du XII' ou du début du XIII' siècle s'en est peut-être fait l'écho lorsqu'elle fait état de chanoines séculiers qui, pour aller vivre désormais comme chanoines réguliers, abandonnèrent leur église détruite par le feu et s'installèrent dans une église nouvelle, construite dans une prairie à l'endroit d'une apparition miraculeuse de saint Martin ${ }^{56}$. Une légende moins ancienne présente SaintMartin comme ayant été à l'origine une simple chapelle dans les prairies basses de l'Ieper(lee) sous le patronage de Notre Dame ou de l'apôtre saint André. En 1012 - peut-être une mauvaise lecture pour l'année 1102, date de la réforme par l'évêque de Thérouanne - elle aurait été consacrée à saint Martin 57.

L'hypothèse que jusqu'à quelques années avant 1102 Saint-Pierre aurait été un chapitre de chanoines séculiers supprimé par l'évêque réformateur Jean de Thérouanne et donc comme tel plus ancien que le chapitre de chanoines réguliers installé par cet évêque dans l'église Saint-Martin en 1 102, va tellement à l'encontre des idées reçues sur l'histoire ancienne d'Ypres, qu'elle sera probablement acceptée difficilement aussi longtemps qu'elle ne repose que sur les indices réunis plus haut et non pas sur des preuves formelles.

Un argument en faveur de l'ancienneté de l'église de Saint-Martin est le patronage de ce saint particulièrement vénéré à l'époque mérovingienne. Beaucoup d'églises situées dans un ancien domaine royal mérovingien ou carolingien avaient saint Martin comme saint patron ${ }^{58}$. Or Ypres avait probablement été, avant de devenir à la fin du $\mathrm{IX}^{\mathrm{e}}$ ou au $\mathrm{X}^{\mathrm{e}}$ siècle une possession du comte de Flandre, un tel fisc royal. L'église d'un domaine se trouvait généralement à proximité du centre d'exploitation de celui-ci, qu'à Ypres nous ne pouvons malheureusement pas localiser, malgré la mention d'un grand centre domanial à Ypres dans le plus ancien compte général du domaine comtal, le «Gros Brief» de $1187^{59}$. Le toponyme Hofland rappelle souvent l'ancienne « réserve» exploitée à partir du centre domanial, mais à Ypres le ressort de la seigneurie comtale du Hofland, intégrée en 1269 par la comtesse Marguerite dans l'échevinage urbain, était tellement étendu, aussi bien à l'est qu'à l'ouest du Grand Marché, que même la grande ferme de quatre hectares appartenant au Zaalhof, non loin de l'église Saint-Pierre, pourrait en avoir fait partie au même titre qu'un hypothétique centre domanial près de Saint$\operatorname{Martin}^{60}$. L'église d'un fisc n'était d'ailleurs pas toujours située aux environs immédiats du centre de celui-ci, comme le montre l'exemple du fisc royal de

56 - PYCKe, « Prévôté Saint-Martin à Ypres...», p. 937 ; CARnIER, «Jan van Waasten... », p. 63, n. 22.

57 - Prcke, «Prévôté Saint-Martin à Ypres...», p. 937 et 938 note 7.

58 - G. Beringis, « Les patronages de saints dans la valléc de l'Escaut », Revue du Nord, t. 69 , 1986, p. 437-439.

59 - VerhUi.ST-GrSSI:LING, Le Compte Général de 1187..., p. 185-187.

60 - DES MAREZ, Elude sur la propriété foncière..., p. 214 et la carte entre p. 216 et p. 217 ; cf. la contribution de O. Mus dans S. VAN Bellingen, M. DewILDE, O. Mus, « De verdwenen SintMichiclswijk te Ieper », Archeologie in Vlaanderen, t. 3, 1993, p. 256-265. 
Marka à l'ouest de Gand, dont l'église se situait à plusieurs kilomètres du centre du fisc ${ }^{(1)}$. Cette piste ne mène donc pas vers une solution concernant l'ancienneté respective des deux principales églises yproises. Celles-ci, en effet, peuvent avoir eu toutes les deux une origine rurale et ancienne si l'on tient compte de la grande extension du domaine royal, puis comtal, à Ypres. L'ancienneté d'une église n'est d'ailleurs pas un argument en faveur de l'ancienneté d'un chapitre séculier dans l'une d'elles. La fondation d'un chapitre séculier au $\mathrm{XI}^{\mathrm{C}}$ siècle a plutôt été fonction de la localisation de l'église concernée près d'une fortification, d'une foire ou d'un autre centre économique, politique ou administratif dont le chapitre pouvait servir les intérêts, matériels et autres ${ }^{62}$. A Ypres ces facteurs jouent aussi bien en faveur de la priorité d'un chapitre à Saint-Pierre qu'à Saint-Martin, aussi longtemps que la localisation de la fortification primitive et de son centre économique ainsi que celle de la foire au début du XII" siècle n'ont pas été établies sur des bases plus solides que celles que nous venons d'indiquer. A cet égard une étude plus poussée de la base du pouvoir à Ypres de Guillaume d'Ypres et de sa localisation dans la ville lors des événements de 1127-1128, à l'aide des récits de Galbert de Bruges et de Walter de Thérouanne, pourrait peut-être apporter encore des éléments nouveaux.

L'explication principale de la naissance de la ville d'Ypres au XI' siècle reste d'ailleurs à nos yeux non pas son rôle ecclésiastique ou politique, mais sa situation géographique. A la lisière d'une région maritime qui grâce à une inondation de la mer connut soudainement et temporairement une production énorme de laine qui ne pouvait être travaillée sur place, une liaison fluviale temporairement facile permettait de transporter celle-ci vers un endroit peuplé de l'intérieur, faisant partie d'un grand domaine rural du comte et situé lui-même au carrefour de la rivière et d'une route terrestre importante qui liait une foire naissante aux autres foires de Flandre en pleine expansion ${ }^{6,3}$.

Adriaan V'ERHUL.ST

Adriaan Vi:RHU.ST, professeur émérite de l'Université de Gand, Mechelsesteenweg 142, Bus 27, B-2018 Anvers.

61 - A. VI:RHII.ST-G. DlichliRCQ. "Gand entre les abbayes el la fortification comtale », dans J. Di: avilit: Gand. Apologie d'une ville rebelle, Anvers. 1989, p. 38.

62 - Di:ClekCQ, «Seculiere kapittels...» (voir plus haut note 48 ).

63 - Cette étude est une version revue et remaniée d’un article que nous avons publié en néerlandais sous Ie titre «Ieper in de $11 \mathrm{e}-12 \mathrm{e}$ ceuw $\gg$ dans De Kerk en de Nederlanden. Archieven. instellingen. samenleving, éd. E.S.C. Erkelens-Buttinger e.a., Hilversum, 1997, constituant des "Mélanges » offerts au professeur C. Dekker de l'Université d'Amsterdam. Le point de départ de notre étude a été une conférence à l'assemblée générale annuelle de la Koninklijke Academie voor Wetenschappen, Letteren en Schone Kunsten van België, tenue à Ypres en 1995. Une grande partie de la bibliographie locale, parfois très difficile à trouver, nous a été signalée et souvent procurée par notre ami J.-M. Duvosquel, maitre de conférences à l'ULB, que nous tenons à remercier vivement. Des discussions avec les érudits locaux $O$. Mus, archiviste honoraire de la ville d'Ypres, et J. Termote, archéologue, nous ont permis de préciser nos vues sur certains points. 


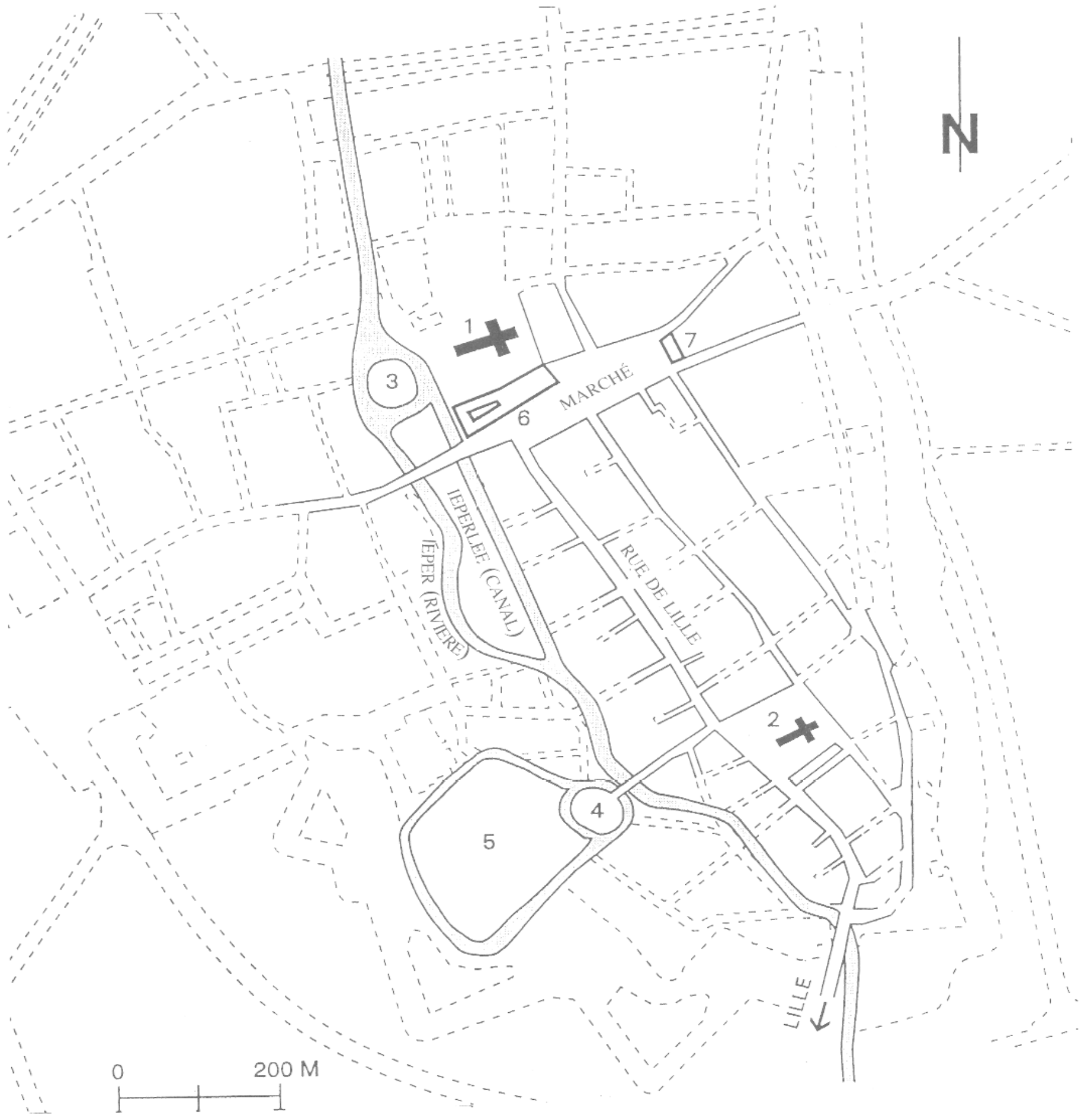

Ypres au XII siècle.

Légende :

1. Eglise Saint-Martin.

2. Eglise Saint-Pierre.

3. Motte circulaire ( $x l^{2}$ siècle).

4. Résidence comtale du «Zaalhof».

5. Manoir du «Zaalhof».

6. Halle aux draps avec beffroi (1173).

7. Hôpital Notre-Dame (1187). 